\title{
Rare diseases and referral centers
}

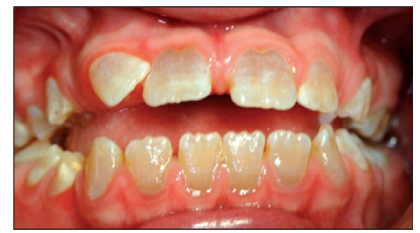

\section{Marie-Laure BOY-LEFÈVRE, Muriel DE LA DURE-MOLLA, Steve TOUPENAY, Ariane BERDAL}

\begin{abstract}
Rare diseases represent a major public health challenge due to their number, presently estimated to be between 6000 and 8000, their severity and the absence of any preventive treatment. The enactment of two National Plans for Rare Diseases by the establishment of Referral Centers and Diagnosis and Treatment Centers has brought about a recognition of the specificity of these disorders, the creation of a epidemiological database, a framework for rapid diagnosis protocols at the first symptoms, greater access to specialized care to accompany the afflicted and their families during their frequently difficult medico-social journey, training of skilled health professionals in the field of these rare diseases, promotion of basic and clinical research and finally the development of European partnerships in both research and therapeutic planning.
\end{abstract}

KEY WORDS

Rare diseases

Syndrome
Dental and orofacial malformations

Referral centers

\section{RARE DISORDERS}

A disorder is considered rare when it affects less than one person in 2000 and in France their number is currently estimated to be around 3 million. More than 7000 rare disorders have been catalogued and between 80 to $85 \%$ are genetic in origin caused by a single gene in the majority of the cases. The responsible genes have been identified for 3000 of them but, for the most part, their functions and the associated physiopathological mechanisms are still unknown. This data was cited from the 
Foundation for Rare Diseases ${ }^{4}$. Research on rare diseases and the development of innovative therapies imply a horizontal approach allowing continuity between basic research, clinical research, diagnosis and care.

Rare diseases are generally very serious, chronic and disabling, often with a potentially fatal diagnosis. They are first manifest in the earliest years of life in more than $50 \%$ of cases and are responsible for more than $30 \%$ of infant mortalities. The complexity of rare disorders is related both to the diversity of its forms and to the progression of the symptoms throughout life of the afflicted and impacts their quality of life. For a large majority of them, there is no preventive therapy.

The ORPHANET ${ }^{7}$ database helps to provide precise information about rare diseases (just type in the name of the disease) for health professionals as well as for those affected and for the public at large.

\section{RARE DISEASES WITH AN OROFACIAL COMPONENT}

Genetic diseases affecting the orofacial region can be either isolated or syndromic. The development of the face, jaws, teeth and their supporting structures forms a biologic continuum (the same for cellular origins, identity of the genes that guide the major morphogenetic steps). These rare congenital malformations variably affect one or several of these structures: skeletal bone, soft tissues, teeth and their supporting tissue including alveolar bone, as well as vascularization and innervation of all of these structures. They do not all express themselves at birth, some of them appear only in the first years of life. More than 1000 pathologies are reported as of today (Table I).

Many large groups of rare orofacial malformations can be identified and correspondingly are due to embryologic clefts, due to quantitative or qualitative structural developmental anomalies, or due to nervous or vascular anatomo-functional anomalies.

One can principally distinguish:

1) facial clefts (labio-palatal, orofacial, lateral, medial) and associated syndromes (Van derWoude, Pierre Robin Sequence, ...);

2) oto-mandibular dysplasias (hemifacial microsomias) and associated

\begin{tabular}{|l|c|}
\hline Region & Number of Syndromes \\
\hline Facial & 1080 \\
\hline Oral & 837 \\
\hline Buccal & 677 \\
\hline Dental & 535 \\
\hline
\end{tabular}

Table I

Number of syndromes affecting the buccal-oro-facial regions according to the LDDB2: (London Dysmorphology Database, Oxford Medical Datbases, Oxford University Press, version 2.2, 2000). 


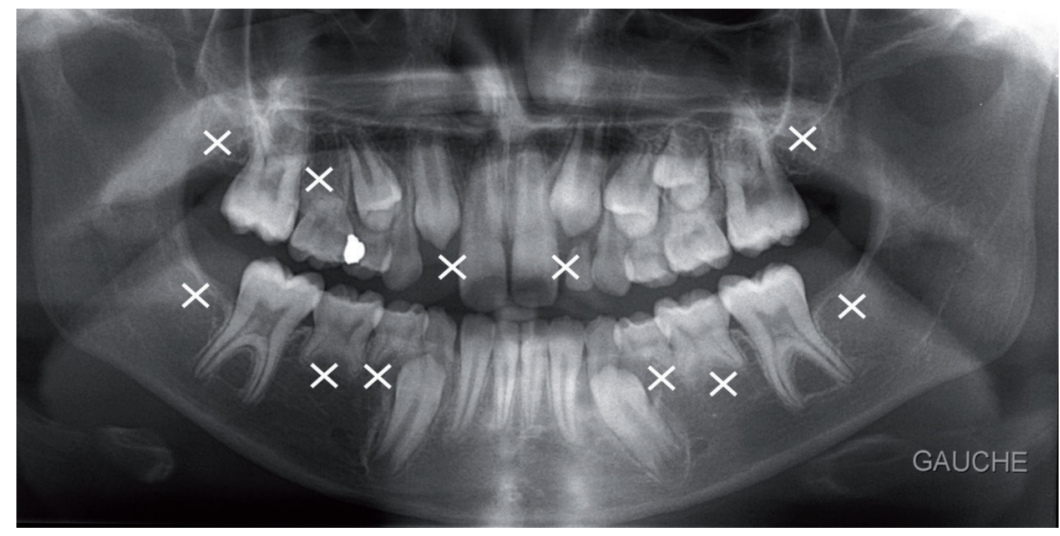

Figure 1

Panoramic radiograph of a patient who presents with an oligodontia.

syndromes (Goldenhar Syndrome (OAV), Treacher-Collins Syndrome, ... );

3) hyper growth dysmorphias;

4) other malformative dysmorphias;

5) paralytic malformations (facial paralysis) and associated syndromes (Moebius Syndrome);

6) vascular malformations and associated syndromes;

7) dental and periodontal malformations either isolated or with associated syndromes.

Whether syndromic or isolated, dental anomalies are grouped into anomalies of number, shape, position, structure and eruption. In the great majority, anomalies of number and of structure are the most common.

\section{Anomalies of number}

Agenesis of more than 6 teeth is rare. Called oligodontia it has a prevalence of $0.14 \%$. It most often affects the permanent teeth and in the most severe cases, both upper and lower arches (Fig. 1). These malformations are frequently accompanied by anomalies of size and shape of the existing teeth as well as alveolar defects associated with skeletal growth problems. Teeth act as guides for the growth and development of the maxillary and mandibular osseous bases. Many genes are known: MSX1, IFR6 and PAX9 whose identification facilitates the search for a wider clinical spectrum and in which the screening can lead to sibling genes. Dental anomalies of number can also accompany a polymalformative or tumor related context, as for example, mutations of the gene AXIN2 that causes in addition to dental agenesis, colorectal cancers.

The functional handicap of this type of pathology is obvious. The loss of 4 molars for example reduces the masticatory coefficient by $40 \%$.

\section{Structural anomalies}

All the dental tissues can be altered. Two isolated pathologies, hereditary amelogenesis imperfecta and hereditary dentinogenesis imperfecta 

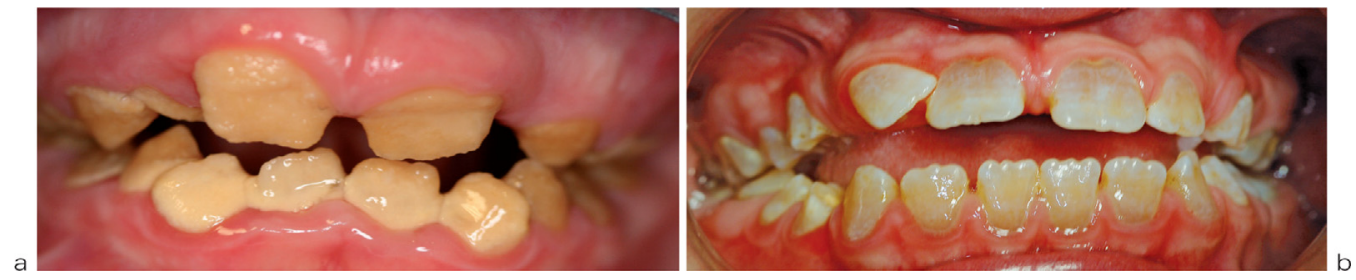

Figure 2

Intra-oral photographs of patients presenting:

a: a structural anomaly of the enamel (hereditary amelogenesis imperfecta);

$b$ : an anomaly of the dentin (hereditary dentinogenesis imperfecta).

have been described to affect respectively the enamel and the dentin (Fig. 2). A few syndromes can also accompany these clinical manifestations.

Progress in molecular genetics diagnosis has demonstrated that, in reality, most structural dental anomalies and anomalies of number constitute a mild form of the syndromes. These very varied and heterogeneous malformations have in common the same requirements for diagnosis' exploration, medical-surgical and dental treatment, and an interceptive functional therapeutic plan during growth. This plan requires a re-evaluation of each treatment step until the end of growth and necessitates intervention by a team formed to treat facial malformations of the oral cavity as well as corresponding surgical treatments. It includes: maxillo-facial surgeons, plastic surgeons, and dentists and all the skillsets of each discipline. Collaboration with other specialties depends on associated signs or syndromes.

Dento-facial orthopedics, orthodontics and dentistry are combined treatments lasting from birth to the end of growth. It may also be indispensible to establish dental and oral rehabilitative treatment that may need to be pursued as a continuum to adulthood.

\section{COMMON PROBLEMS OF RARE DISEASES}

EURORDIS, federation of associations of diseases and of individuals active in the domain of rare diseases, in 2005 listed different common factors of rare disorders affecting patients and their relatives. These include the absence or delay in making the correct diagnosis, the lack of scientific knowledge, the lack of unequal access to care, the inherent social consequences, and the high cost of some medical devices and some medications when they exist ${ }^{3}$.

\section{Misdiagnosis}

Diagnosis is often made late due to the variation of the course of the disease. The period between the appearance of the first symptoms or clinical signs and the appropriate 
diagnosis can cause important delays and a lost opportunity to start treatment $^{8}$. In addition, some erroneous diagnoses can lead to inappropriate or even iatrogenic treatment.

\section{Insufficient patient's information}

There often exists a lack of patient information both about the disease and the possible medical and socioeconomic help that is available. The identification of less frequent cases by health professionals has also proven equally difficult.

The role of associations for affected patients at the national and European level is crucial and helps to improve communication between the families and the health care professionals.

\section{Complexity of the therapeutic methods and the courses of care}

The therapeutic strategy is not always simple in the medium to longterm. In this respect, the establishment of national protocols of diagnosis and care (NPDC) must also contribute to the establishment ofprotocols for specific therapeutic care. The course of care is also often complex due to the number of medical, paramedical and dental disciplines that are involved in the treatment plan. Access to care and to qualified professionals is difficult, because it might possibly be necessary to mobilize a group of specialists.

\section{Impact on the quality of life and on social integration}

A rare disease has repercussions that affect the quality of life, often translating into an embarrassment (functional, esthetic), a more or less intense pain, a psychological discomfort, a loss of confidence or even the onset of a disability, a handicap of varying severity. Its consequences affect all facets of life: relationships, school, choice of type of work, leisure activities as well as emotional life ${ }^{9}$. It can lead to stigmatization, isolation, even exclusion from the social community. These repercussions can weigh more heavily if many family members are similarly afflicted.

\section{Financial impact}

This point is included in our scope of activities because of the high cost and for the remaining charges to the patient for the complex dental rehabilitation involving the placement of implants in the cases of multiple dental ageneses or of crowns and veneers in the cases of amelogenesis imperfecta. It must be also be made clear that despite the context of being a rare disease, there is no special orthodontic method of treatment for these patients while it is inevitably more complex in the vast majority of such cases.

The only singular method of treatment established by the CNAM concerns implant treatment for these patients, children and adults, presenting with a rare oligodontia (Fig. 3). 


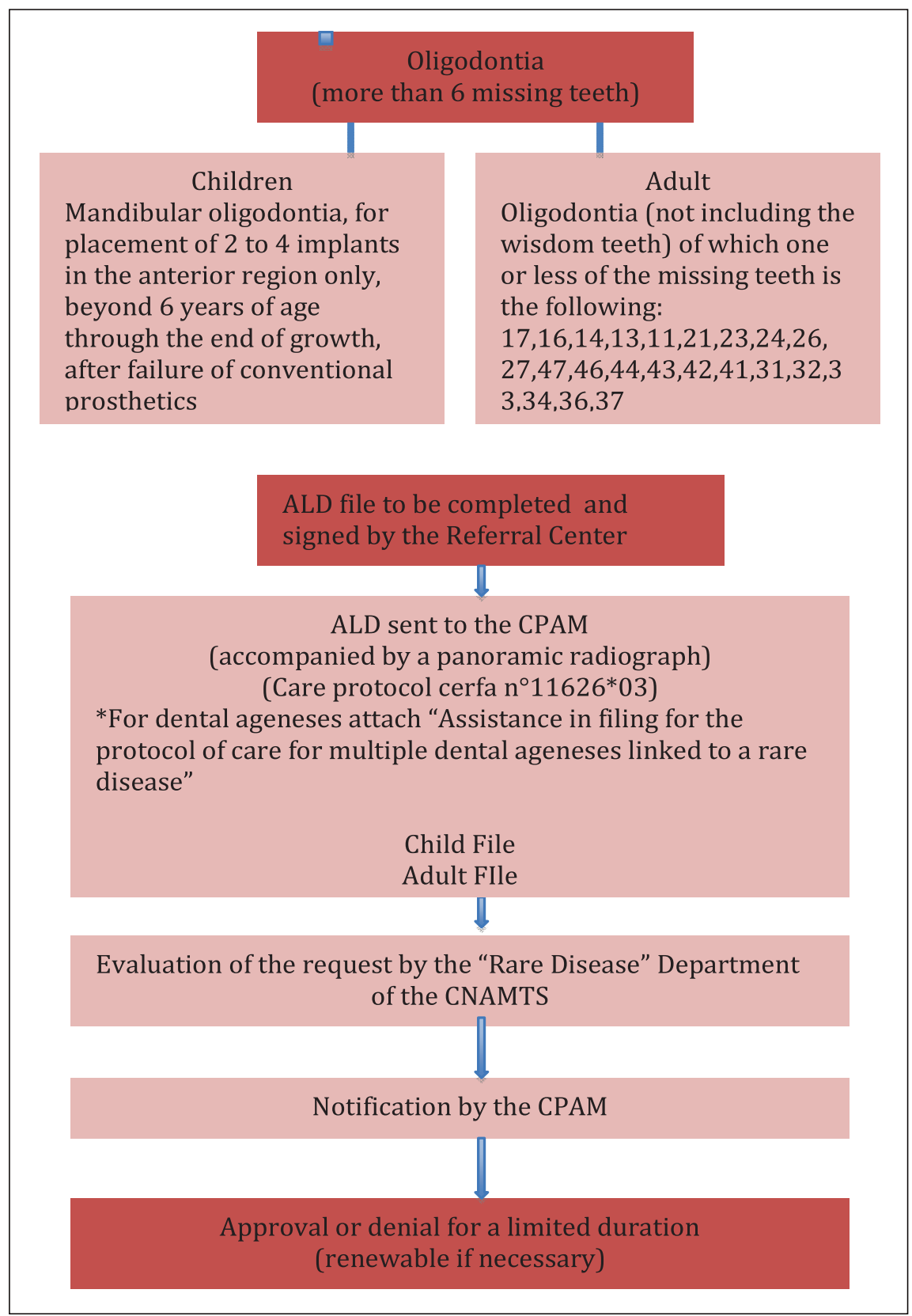

Figure 3

Application of the nomenclature for codes and prices registered at the NGAP.

\section{RARE DISEASES, A MAJOR PUBLIC HEALTH ISSUE}

Rare diseases have been considered one of five great priorities of the law relative to the politics of the Pub- lic Health Law of 9 August, 2004 of the first National Plan for Rare Diseases (PNMR 20050-2008) "Assuring 

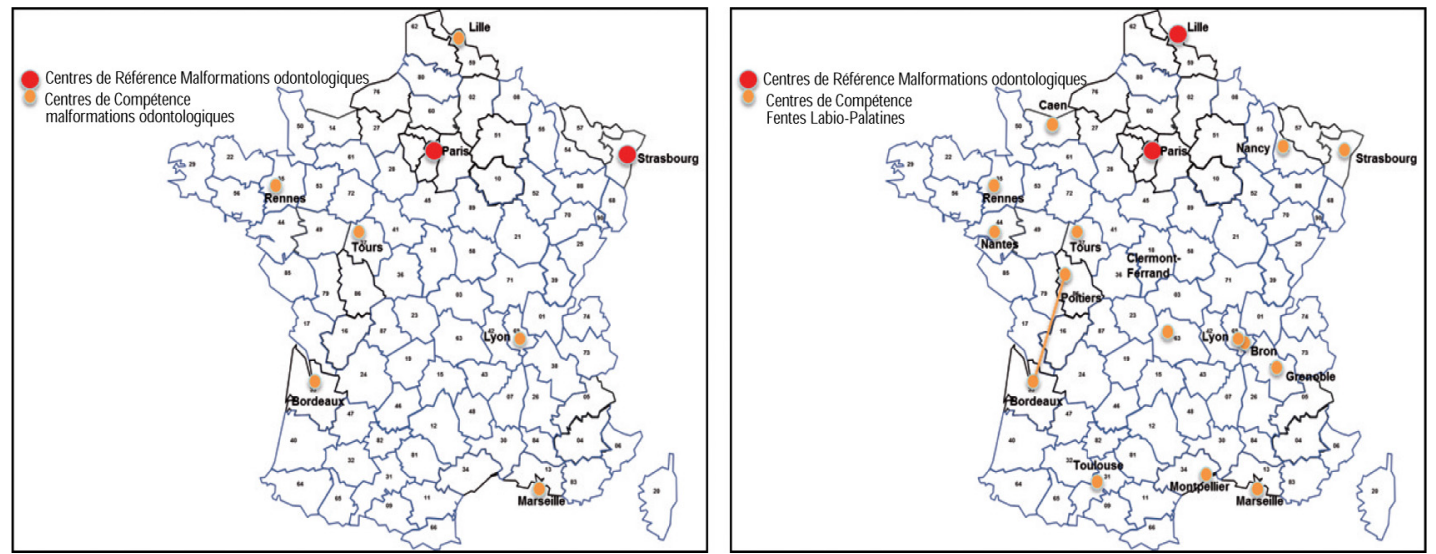

Figure 4

National distribution of Referral Treatment Centers for maxillo-facial surgery and orthodontics.

equality for access, to diagnosis procedures, to treatment and to support $^{6 "}$ which proposed a series of concrete measures and structures in order to organize the provision of care in France and to make it more easily understood by patients:

- initially, the structures of scientific and clinical excellence for rare diseases were identified and recognized through a process of designating Referral Centers for one (or a group) of rare disease(s); these Referral Centers for rare diseases assure both a level of expertise with specific competence and knowledge for a disease or a group of rare diseases and provide a recourse to expertise beyond its location.

- then, progressively, around these Referral Centers, a network of specialized care formed to improve access to diagnosis and to quality of support, with the identification of Skilled Centers (regional or interregional) for rare diseases that justified its establishment.
The missions of the Referral Centers have been defined as follows ${ }^{1}$ :

- to facilitate the diagnosis and to define a strategy of supported therapeutic strategies, psychological and social support;

- to define and disseminate the supported treatment protocols, in connection with the High Authority of Health (HAS) and the national union of sickness insurance funds (UNCAM);

- coordinate research and participate in epidemiological surveys, in connection with the national institute of health watch (INVS);

- to participate in training and information for health professionals, the patients and their families, in connection with the national institute of prevention and education for health (INPES);

- lead and coordinate the networks of health and medico-social correspondents;

- be the designated trustees for the public bodies and associations for the afflicted. 


\section{First consultation at the Multidisciplinary Referral Center}

- Radiographic evaluation (panoramic, profile Cephalometric)

- History familial and medical

- Exact phenotype analysis

- +/- Prescription from the complete examination

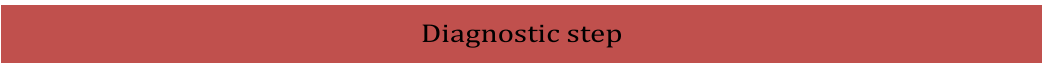

\section{Simple Type}

Syndromic Type

Direction towards other

Referral Centers or

Referral Centers or
specialized Services

Diagnostic position. Not sure

\section{Strategic supported therapies}

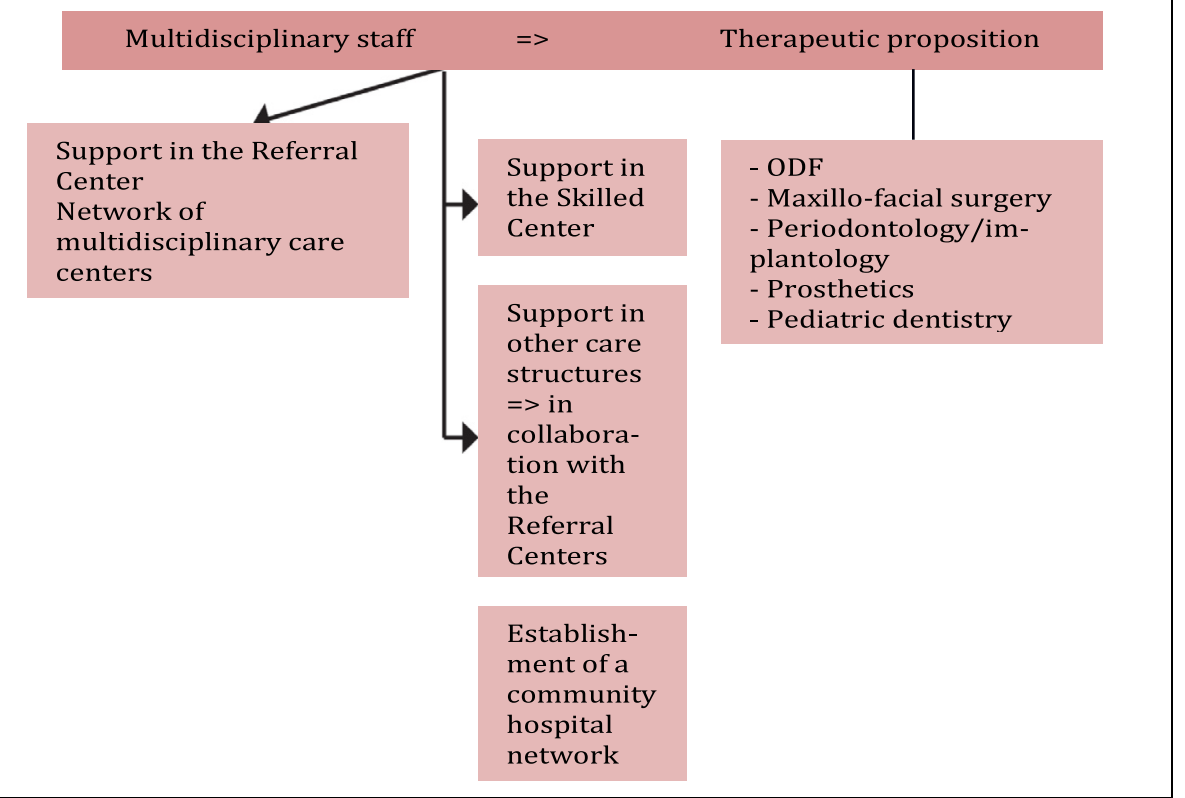

Figure 5

Course of diagnostic and therapeutic support of patients with rare diseases.

More recently, a second Plan for Rare Diseases has been established for the period 2011-2014 "Quality of care, research, Europe: a renewed ambition" 6 . The National health policy helped to develop a genuine source for treatment for the afflicted with the designation of 134 Certified Referral Centers (CRMR) each dedicated to the treatment of one or a 
group of related diseases. These CRMR are linked to a network of more than 500 Skilled Centers ${ }^{4,7}$.

Two Referral Centers are focused on orofacial and dental malformations and were certified in 2006 and 2007:

- Referral Center for dental signs of rare diseases (Coordinator: Pr. Marie-Cecile Maniere) Service d'odontologie des hopitaux de Strasbourg;

- Referral Center for rare malformations of the face and of the oral cavity (Coordinator: Pr. Marie-PauleVazquez,) Service de chirugie maxilla-faciale de I'hopital Necker et Pr Ariane Berdal, coordination pour the Service d'odontologie de I'hopital Rotschild, AP-HP.

Therefore there is a genuine national network of Referral and Maxillofacial and Dental Expertise Centers in France (Fig. 4).

It is, however, clear that the proximity between the patient and the treating doctor can but only improve the therapeutic management. This is the why the objective for each Center for Expert Care has as its objective to create a genuine "Open Healthcare Network". The Referral Centers are multidisciplinary in terms of both diagnosis and therapy. Treatment plans are effectively discussed by the staff leaving the treating practitioner to put the therapies to work while benefitting from the support and the expertise of the CR's (Fig. 5).

The short-term objective is to focus on and reinforce the relations between the Referral Centers, Centers of Expertise and local practitioners with the establishment of a network of community hospitals in order to optimize the course of treatment and to support patients starting from a young age.

Conflicts of interest: none

\section{BIBLIOGRAPHY}

1. Un centre de référence : c est quoi ? 21 janvier 2013. http://www.sante.gouv.fr/ un-centre-de-refe-rence-c-est-quoi.html

2. London Dysmorphology Database, Oxford Medical Databases, Oxford University Press, version 2.2, 2000.

3. "Maladie rare : comprendre une priorité de santé publique», Novembre 2005, EURORDIS, wWw.eurordis.org

4. Les Maladies rares, définitions. http://fondation-maladiesrares.org/definitions

5. Molla M, Bailleul-Forestier I, Artaud C, Verloes A, Naulin-Ifi C, Elion J, Berdal A. Odontogénétique, EMC 2005;28-090-M610.

6. Plan National Maladies Rares. http://fondation-maladiesrares.org/plan-national-maladiesrares

7. Le portail des maladies rares et des médicaments orphelins. www.orphanet.fr

8. Toupenay S. La prise en charge des maladies rares en odontologie : un enjeu de santé publique. Thèse Dipl. Doct. Paris : Univ. D Diderot, 2010.

9. Toupenay S, Razanamihaja N, Berdal A, Boy-Lefèvre ML. Rare diseases with oral components: care course and quality of life. Community Dent Health 2013;30(1):10-4. 